\title{
Idebenone - a new drug for patients with Leber's hereditary optic neuropathy
}

\author{
Dorota Szumny ${ }^{1,2}$, Karolina Czajor ${ }^{2,3}$, Małgorzata Mulak ${ }^{2,3}$ \\ ${ }^{1}$ Chair and Department of Pharmacology, Medical University of Wroclaw \\ Head: Prof. Adam Szeląg, MD, PhD \\ ${ }^{2}$ Department of Ophthalmology, University Clinical Hospital in Wroclaw \\ Head: Prof. Marta Misiuk-Hajło, MD, PhD \\ ${ }^{3}$ Department and Clinic of Ophthalmology, Wroclaw Medical University \\ Head: Prof. Marta Misiuk-Hajło, MD, PhD
}

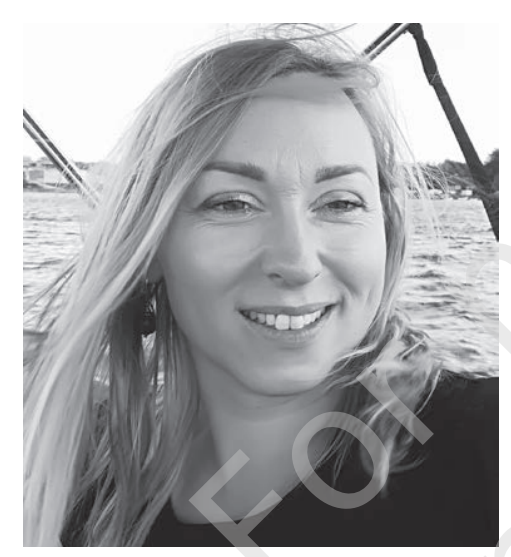

H I GHLIGHTS

Idebenone is a new drug in the treatment of Leber's hereditary optic neuropathy.

\begin{abstract}
Leber's Hereditary Optic Neuropathy is a rare disease caused by a mutation in the mitochondrial DNA. It appears most often in young men, leading to profound, permanent loss of vision in a short time. There is no specific treatment for this condition.

Idebenone is a medicine that administered to patients with Leber's hereditary optic neuropathy improves or stops the deterioration of vision. Increasing the number of people during therapy and the duration of treatment will allow in the future to answer the question, whether this drug is effective and safe in a larger group of patients.
\end{abstract}

Key words: idebenone, Leber hereditary optic neuropathy, LHON 


\section{INTRODUCTION}

Leber's hereditary optic neuropathy (LHON) is a rare disease entity with a minimum prevalence estimated to be $1: 10$ 000. However, epidemiological studies in different regions have shown large variations in the incidence of the disease, e.g., in the northern part of the UK, the incidence is $1: 31$ 000, and in Finland, it is $1: 50000$ [1]. The course of the disease results in an acute or subacute, painless loss of central vision, initially in one eye and later (within weeks or months) in the other eye. It manifests predominantly in men between the ages of 20 and 30, although it can also occur in younger people, people over the age of 70 , and in women of all ages. Central or centrocecal visual field loss, impaired color vision in the red-green axis, and decreased contrast sensitivity are observed. Although initially one eye is usually affected (damage to the other eye occurs after an average of 2-6 months), in $25 \%$ of cases the disease begins bilaterally.

LHON is one of the most common mitochondrial diseases. It is caused by a point mutation within the mitochondrial DNA (mDNA), leading to a defect in the respiratory chain complex as a result of changes in the genes encoding the subunits of the complex. Approximately 95\% of LHON cases are responsible for one of the following three mDNA mutations: $3460 G>\mathrm{A}, 11778 \mathrm{G}>\mathrm{A}$, and $14484 T>\mathrm{C}$ [1-4]. Mitochondria with the mDNA are inherited almost exclusively in the female line, so the mutated gene is passed from the affected mother to her children regardless of gender. The incidence is much more common in males, and the clinical picture of the disease can vary greatly due to the degree of heteroplasmy, that is, the simultaneous occurrence of mutant DNA molecules alongside mutation-free mDNA in a single cell [5]. Mutant and normal mitochondria are passed on to offspring, and the manifestation of full-blown Leber syndrome and its severity occurs with significant levels of mutant mDNA. Pathological changes in the mitochondria are not sufficient for disease development; mutations in the cell nucleus are probably also required. The variability of symptoms in individuals suggests the influence of still other, extrinsic factors, such as alcoholism, nicotinism, $B$ vitamin deficiency, and a morphologically small nerve II disc $[5,6]$. It should be noted that vision loss occurs in $50 \%$ of male carriers of the defective gene, and only in $10 \%$ of females, which is explained by the protective effect of female sex hormones [4-6].

Ophthalmoscopic examination in the early stages of the disease may not reveal pathology - the fundus of the eyes may be normal, which applies to $20-40 \%$ of cases. In the early stages of the disease, dilatation or tortuosity of periorbital vessels, and congestion and swelling of the disc with subsequent fading starting from the temporal part can be observed, which usually occurs after about 6 weeks. The initial normal fundus picture can make diagnosis dif- ficult and cause delays, so in doubtful cases, it is worthwhile using additional tests such as ganglion cell complex (GCC) and/or periventricular fiber (RNFL) testing, which can show abnormalities even in asymptomatic carriers. Reduction in the GCC complex and thinning of the perifoveal fibers may precede fading of the optic disc. Electrophysiologic studies (pattern electroretinogram [PERG], multifocal electroretinogram [mfERG], and visual evoked potentials $[\mathrm{VEP}])$ may be helpful in unclear cases. Genetic testing is the conclusive test [5-7].

Sometimes, ophthalmic symptoms are accompanied by osteoarticular, cardiac (arrhythmia), or neurological pathologies such as neuropathy, dystonia, dysarthria, or myopathy. The coexistence of extraocular lesions is referred to as Leber-Plus disease $[1,5]$.

In the absence of specific treatment, the prognosis for vision is not favorable. Supportive treatment with folic acid or a combination of B vitamins has been proposed. In the 2016 recommendation of the Haute Autorité de Santé (HAS), lifestyle interventions consisting of quitting smoking and alcohol consumption were suggested. Furthermore, the use of antioxidants (glutathione, vitamin E, and coenzyme Q10) were recommended as a treatment. The positive effect of steroid therapy, which can also be evaluated in terms of protection the fellow eye, has not been demonstrated. This prompts the search for new therapeutic methods [8].

\section{IDEBENONE}

In September 2015, idebenone received European Medicines Agency registration for the treatment of visual impairment in children over 12 years of age and adults with LHON. Reimbursement for the treatment is provided in Germany, Sweden, Norway, Luxembourg, and under a special program in France.

Idebenone is a short-chain benzoquinone, a synthetic analogue of coenzyme Q-10. As a result of its hydrophobic structure, it passes through mitochondrial membranes. It has antioxidant activity and protects cells from oxidative damage.

The mechanism of idebenone action includes stimulation of ATP biosynthesis, increasing the antioxidant potential of cells, and removing free radicals. Furthermore, idebenone protects cell membranes from lipid peroxidation by bypassing complex I of the mitochondrial respiratory chain [9]. Idebenone may thus improve the production of high-energy compounds by the respiratory chain in the presence of mitochondrial complex I damage.

Attempts are being made to use idebenone to treat Alzheimer's disease and Duchenne muscular dystrophy. The drug has also been used to treat Huntington's disease, mitochondrial encephalomyopathies, and Friedreich's ataxia, but the results in this regard are inconclusive. Moreover, 
idebenone has been used topically to reduce wrinkles and improve skin quality in middle-aged women, though this effect also needs further investigation [10].

According to the results of the current study, idebenone used in the treatment of LHON prevents further vision loss and promotes improved vision compared to the natural course of the disease.

The efficacy of idebenone treatment, among others, was evaluated in a randomized, controlled 24-week clinical trial: RHODOS (Rescue of Hereditary Optic Disease Outpatient Study). The drug was administered at $900 \mathrm{mg} / 24 \mathrm{~h}$ in three individual doses. Patients aged 14-64 years who carried mDNA mutations (G11778A, G3460A, T14484C) were studied. The study included 53 patients who received the drug and 29 who received placebo. The results of the study demonstrated an improvement in visual acuity in the study group as compared to the control group, but the improvement was not statistically significant. A clinically significant recovery was observed (e.g., reading two more lines on the visual acuity test board than before the drug, or reading one line for patients who had not previously read any letter on the board). As a result of the study, idebenone was found to prevent further vision loss. A greater improvement in vision was observed when treatment was started early in the course of the disease (within 1 year of the appearance of the first symptoms). Idebenone may also protect against color vision loss, which is one of the first symptoms of LHON. Variability in treatment outcomes depending on the type of mutation was also noted. Data on the safety and efficacy of long-term idebenone therapy for patients with LHON are expected after the completion of the phase IV clinical trial: LEROS (External Natural History Controlled, Open-Label Intervention Study to Assess the Efficacy and Safety of Long-Term Treatment With Raxone in Leber's Hereditary Optic Neuropathy), which began in 2016.

Idebenone should be administered with food, as its bioavailability is then significantly increased. Side effects are rare and may include nausea, vomiting, abdominal pain, loose stools, rapid heartbeat, and an increased risk of infection. The interactions of idebenone are not known, and the dosage may vary depending on the indication.

In 2016, an international group of experts established recommendations for the use of idebenone in the treatment of LHON. Patients with this disease should be treated within the first year of symptom onset in the other eye with idebenone at a dose of $3 \times 300 \mathrm{mg}$ daily for at least 1 year, and treatment should be continued in patients who show a response to treatment until a sustained plateau of 1 year is reached [11].

The introduction of a new drug offers an opportunity to improve patients' quality of life. However, there are several questions to be answered that require further clinical studies.

\section{What is the ideal duration of treatment?}

A longer course of treatment with idebenone may offer additional therapeutic benefits and may lead to significant improvements in vision, even if the disease is established and the patient has severe vision loss at the time of treatment initiation [12]. In a large retrospective study, a determinant of better prognosis for visual improvement was early treatment initiation and a longer course of treatment [13]. The mean duration of treatment was approximately 17 months after the initiation of idebenone therapy [14].

\section{What is the maximum time window from the onset of symptoms to the initiation of idebenone therapy?}

Idebenone therapy is likely to be most effective when started early, as retinal ganglion cell loss is minimal early in the disease [12]. There is currently no solid evidence to support the use of idebenone in patients with long-term vision loss, but this requires further study.

\section{Is the therapy safe for children and people taking medications for other diseases?}

LHON can manifest in children younger than 10 years of age [15] and in older patients with late visual loss [16], who have multiple comorbidities and a polypharmacy issue. For both groups of patients, robust data on the efficacy and safety of idebenone are needed. Published data on the treatment of children and elderly patients with LHON are mainly based on single case reports [14].

\section{Is it possible to monitor therapy?}

An increase in the safety of therapy may follow the identification of a biomarker to predict the response to treatment. Currently, no biomarker with a similar function is available. The studies to date involve the use of idebenone in symptomatic patients with a deterioration in visual acuity. We have no data on the use of idebenone as prophylaxis in asymptomatic LHON mutation carriers.

\section{Is the drug effective in patients with additional non- -ocular symptoms?}

Anecdotal data on the use of idebenone in patients with LHON-plus have been published [17-19] but they are insufficient to assess the possible effect of idebenone on extraocular manifestations of the disease. The same is true for the effect of idebenone in patients with multiple sclerosis (LHON + multiple sclerosis) (Harding's disease) [20, 21].

\section{What are the potential directions for therapy development?}

The mDNA copy number may be a determinant of disease conversion in LHON mutation carriers, and affecting mitochondrial biogenesis is a potential therapeutic strategy for further evaluation [22]. Recently, it has been shown that 
idebenone may affect mitochondrial biogenesis under specific circumstances [23], but further studies are needed to clarify this observation.

Clinical trials on idebenone for the treatment of LHON are also difficult as a result of the rare occurrence of the disease. Results from the available studies suggest an improvement or stabilization of vision in treated patients as compared to patients not receiving the drug, who experience rapid deterioration of vision leading to profound vision loss.

\section{CONCLUSIONS}

Since the introduction of idebenone to the market, patients have been treated in many centers around the world. There is a significant increase in the treatment population and duration of treatment, which will likely, over time, allow us to accurately answer some of the above questions and evaluate the efficacy of the therapy in a larger group of patients.

\section{CORRESPONDENCE \\ Dorota Szumny, MD, PhD \\ Katedra i Zakład Farmakologii, \\ Uniwersytet Medyczny we Wrocławiu \\ 50-345 Wrocław, Mikulicza-Radeckiego 2 \\ e-mail: dorotaszumny@wpl}

\section{ORCID}

Dorota Szumny - ID - http://orcid.org/0000-0002-7814-3517

Karolina Czajor - ID - http://orcid.org/0000-0002-4230-6134

Małgorzata Mulak - ID - http://orcid.org/0000-0003-3598-6693

\section{References}

1. Berardo A, Emmanuele V, Vargas W et al. Leber hereditary optic neuropathy plus dystonia, and transverse myelitis due to double mutations in MT-ND4 and MT-ND6. J Neurol. 2020; 267(3): 823-9.

2. Majander A, Bowman R, Poulton J et al. Childhood-onset Leber hereditary optic neuropathy. Br J Ophthalmol. 2017; 101(11): 1505-9.

3. Priglinger C, Klopstock T, Rudolph G et al. Leber'sche hereditäre Optikusneuropathie. Klin Monbl Augenheilkd. 2019; $236(11): 1271-82$.

4. Yu-Wai-Man P, Votruba M, Moore AT et al. Treatment strategies for inherited optic neuropathies: past, present and future. Eye (Lond). 2014; 28(5): 521-37.

5. Roskal-Wałek J, Gierada M, Mackiewicz J. Dziedziczna neuropatia wzrokowa Lebera - opis przypadku. Klinika Oczna / Acta Ophthalmologica Polonica. 2018; 120(4): 227-31.

6. Piotrowska-Nowak A, Krawczyński MR, Kosior-Jarecka E et al. Mitochondrial genome variation in male LHON patients with the m. 11778G > A mutation. Metab Brain Dis. 2020; 35(8): 1317-27.

7. Wang M, Guo H, Li S et al. Electrophysiological and Structural Changes in Chinese Patients with LHON. J Ophthalmol. 2020; 2020: 4734276.

8. Newman NJ. Treatment of hereditary optic neuropathies. Nat Rev Neurol. 2012; 8(10): 545-56.

9. Suno M, Nagaoka A. Inhibition of lipid peroxidation by idebenone in brain mitochondria in the presence of succinate. Arch Gerontol Geriatr. 1989; 8(3): 291-7.

10. Lyseng-Williamson KA. Idebenone: a review in Leber's hereditary optic neuropathy. Drugs. 2016; 76(7): 805-13.

11. Carelli V, Consensus Study Group. Consensus on guidelines for idebenone administration in Leber's hereditary optic neuropathy (LHON). Acta Ophthalmol. 2016; 94.

12. Klopstock T, Metz G, Yu-Wai-Man P et al. Persistence of the treatment effect of idebenone in Leber's hereditary optic neuropathy. Brain. 2013; 136(2): e230.

13. Carelli V, La Morgia C, Valentino ML et al. Idebenone treatment in Leber's hereditary optic neuropathy. Brain. 2011; $134(9):$ e188.

14. Mashima Y, Hiida Y, Oguchi Y. Remission of Leber's hereditary optic neuropathy with idebenone. Lancet. 1992; 340(8815): 368-9.

15. Barboni P, Savini G, Valentino ML et al. Leber's hereditary optic neuropathy with childhood onset. Invest Ophthalmol Vis Sci. 2006; 47(12): 5303-9.

16. Dimitriadis K, Leonhardt M, Yu-Wai-Man P et al. Leber's hereditary optic neuropathy with late disease onset: clinical and molecular characteristics of 20 patients. Orphanet J Rare Dis. 2014; 9(1): 158.

17. Carelli V, Valentino ML, Liguori R et al. Leber's hereditary optic neuropathy (LHON/11778) with myoclonus: report of two cases. J Neurol Neurosurg Psychiatry. 2001; 71(6): 813-6. 
18. Watanabe M, Mita S, Takita T et al. Leber's hereditary optic neuropathy with dystonia in a Japanese family. J Neurol Sci. 2006; 243(1-2): 31-4.

19. Nikoskelainen E, Marttila RJ, Huoponen K et al. Leber's "plus": neurological abnormalities in patients with Leber's hereditary optic neuropathy. J Neurol Neurosurg Psychiatry. 1995; 59(2): 160-4.

20. Harding A, Sweeney MG, Miller DH et al. Occurrence of a multiple sclerosis-like illness in women who have a Leber's hereditary optic neuropathy mitochondrial DNA mutation. Brain. 1992; 115(4): 979-89.

21. Cortelli P, Montagna P, Pierangeli G et al. Clinical and brain bioenergetics improvement with idebenone in a patient with Leber's hereditary optic neuropathy: a clinical and 31P-MRS study. J Neurol Sci. 1997; 148(1): 25-31.

22. Bianco A, Martínez-Romero I, Bisceglia L et al. Mitochondrial DNA copy number differentiates the Leber's hereditary optic neuropathy affected individuals from the unaffected mutation carriers. Brain. 2016; 139(1): e1.

23. Augustyniak J, Lenart J, Zychowicz M et al. Mitochondrial biogenesis and neural differentiation of human iPSC is modulated by idebenone in a developmental stage-dependent manner. Biogerontology. 2017; 18(4): 665-77.

Authors' contributions:

Dorota Szumny: research concept and design, data acquisition, data analysis and interpretation, editing the article, critical corrections in terms of important intellectual content, final approval of the version to be submitted;

Małgorzata Mulak: research concept and design, data acquisition, final approval of the version to be submitted;

Karolina Czajor: data acquisition, data analysis and interpretation, critical corrections in terms of important intellectual content, final approval of the version to be submitted.

Conflict of interest:

None.

Financial support:

None.

Ethics:

The content presented in the article complies with the principles of the Helsinki

Declaration, EU directives and harmonized requirements for biomedical journals. 